Financial Crime Update

Iwa Salami reviews the latest financial crime developments

Since the launch of bitcoin in 2009, there have been fierce calls for the regulation of cryptocurrencies particularly linked with, amongst other things, their use to facilitate financial crime. 0ne of the mechanisms deemed best to achieve this has been at the point of their intersection with mainstream finance - which is mostly through cryptocurrency exchanges and wallet providers which convert fiat currency to cryptocurrency and vice versa. The Financial Action Task Force (FATF)

Recommendation 16 is expected to achieve this, however, this rule is only as strong as its robust implementation globally. The growth of decentralised finance (DeFi) further exacerbates the challenges of regulating the crypto industry, particularly as it is not covered by FATF Recommendation 16.

\section{DECENTRALISED FINANCE: THE CASE FOR A WHOLISTIC APPROACH TO REGULATING THE CRYPTO INDUSTRY}

\section{While FATF Recommendation 16 applies to centralised} exchanges and wallet providers (custodial exchanges and wallet providers) it does not apply to decentralised platforms for crypto transactions (non-custodial finance). This article considers, first, the potential limitations of the FATF rules with respect to centralised exchanges. It then examines the limitations in the context of decentralised finance. It assesses the risk of $\mathrm{DeFi}$, necessitating regulation. It then highlights the policy issues around the regulatory gaps and concludes with suggestions for a wholistic approach to regulating the crypto industry.

\section{FATF RECOMMENDATION 16 AND CENTRALISED AND DECENTRALISED PLATFORMS}

FATF Recommendation 16 introduced travel rules for virtual assets service providers (VASPS) which are similar to the travel rules for international banks. The FATF Recommendations document defines VASPS as:

“... any natural or legal person who ... as a business conducts one or more of the following activities or operations for or on behalf of another natural or legal person:

\footnotetext{
(i) exchange between virtual assets and fiat currencies;

(ii) exchange between one or more forms of virtual assets;

(iii) transfer of virtual assets;

(iv) safekeeping and/or administration of virtual assets or
}

instruments enabling control over virtual assets; and

(v) participation in and provision of financial services related to an issuer's offer and/or sale of a virtual asset."

Recommendation 16 requires that transfers of more than US $\$ 1,000$ must contain information from both the sender and the recipient and be passed on to the receiving institution. With this travel rule, VASPS including crypto exchanges must therefore exchange data between payment senders and recipients (being natural or legal persons) as an Anti-money laundering / Know-Your-Customer (AML/ $\mathrm{KYC}$ ) requirement.

Although FATF applies to activities and operations conducted on all centralised exchanges and through custodial wallets, its effectiveness is dependent on its effective application globally, so it is only as effective as its effective implementation in all countries where VASPs including cryptocurrency exchanges and wallet providers operate. If countries have weak regulatory regimes or have a weak framework for enforcing AML/KYC requirements, those jurisdictions can facilitate regulatory arbitrage as users intent on committing financial crime would be inclined to transact/operate from those jurisdictions to avoid stringent regulation. So, although the implementation deadline for FATF Recommendation 16 is June 2020, the implementation of this provision is likely to be faced with challenges.

Also, although FATF Recommendation 16 applies to VASPS - businesses which provide financial services for users, including centralised cryptocurrency exchanges and wallet providers or custodial exchanges and wallet providers - Recommendation 16 is not applicable to providers of decentralised exchanges which provide a platform for users to engage in financial and banking services such as loans, earning interest on deposits, directly with other users and on a peer to peer basis without an intermediary (such as a bank) and replacing that intermediary with a smart contract. These are crypto to crypto transactions and users in the DeFI space only need the private keys to their cryptocurrency wallet to be able to transact. A "crypto to crypto" transaction removes the requirement for a natural or legal person to act on behalf of another natural or legal person. (See paragraph on "Limitations of DeFi" overleaf for further explanation).

\section{WHAT IS DECENTRALISED FINANCE (DeFi)}

$\mathrm{DeFi}$ (also known as non-custodial finance) provides the same financial services without any traditional central authority or intermediaries. 
The DeFi ecosystem is built on: blockchains (which store digital information within an immutable, trusted and distributed network), cryptocurrencies, and smart contracts. Promoters of DeFi argue that it enables anyone in the world to engage with a range of financial services including payments, lending, borrowing, and investing without intermediaries. Developers are able to create DeFi decentralised (finance) applications (DApps) on blockchains for any of these purposes. Smart contracts self-enforce agreements between parties creating a transparent and functioning financial system without intermediaries.

Of the numerous DeFi services the fastest growing one is lending and borrowing, also known as cryptocurrency/crypto lending. Cryptocurrency lending is a person-to-person lending, which matches borrowers and lenders directly. The lender provides a loan and the borrower has to pay back the borrowed cryptocurrency. Usually a crypto loan is collateralised by cryptocurrency assets and secured through a smart contract. The length of the loan can range from a few days to months, and even years. The interest rate is based on the amount of cryptocurrency the borrower uses as collateral. Crypto lending platforms accept different types of cryptocurrency as collateral and give the borrowers either cash or cryptocurrency in return.

The largest lending DeFi provider is MakerDAO. MakerDAO is a decentralised application that runs entirely on the Ethereum blockchain and automated smart contracts. It allows users to lock Ethereum (ETH) into a smart contract as collateral to secure loans in its stablecoin called DAI. (Stablecoin are cryptocurrencies designed to minimise the volatility of the price of the stable coin relative to a stable asset or basket of assets). For each DAI, there is $\$ 1.5$ of ETH locked into the MakerDAO smart contract as collateral. If the amount of collateral falls below $150 \%$, then the smart contract is auto-liquidated with a $13 \%$ forced-liquidation fee plus the annual stability fee (annual interest rate for the loan). Its main attraction is that DAI's value is pegged to the US dollar, and it always stays around $\$ 1$, due to its dual coin system, Maker (MKR) and Dai (DAI), which brings price stability to crypto assets. Additionally, it provides liquidity to ETH holders. So, while retaining ownership of ETH holders have access to a liquid and non-volatile fund (DAI) for trading cryptocurrency with leverage, or other purposes. If the price of ETH increases during the loan period, the collateral providers' collateral will increase in value as well, so they would have earned more from their ETH used as collateral after they paid off their debt.

\section{BENEFITS OF DeFi}

Supporters of cryptocurrency lending argue that it facilitates financial inclusion and access to credit as it allows anyone to obtain a loan. This can benefit small-to-medium sized enterprises and individuals without a credit history as it does not utilise credit scoring and also does not require the fulfilment of KYC rules - which inhibit access to finance in formal banking systems. Nonetheless, as cryptocurrencies are volatile, almost all crypto loans are overcollateralised in order to act as a safety net for lenders. As such, the main factor in accessing a loan in crypto lending is how much crypto a borrower is able to put up as collateral. In general, crypto lending terms require collateral ratios of $150 \%+$ to secure a loan, which provides a safety net for lenders to manage risk especially as there is no credit score involved in the evaluation process. With this amount of collateral though, it is arguable whether cryptocurrency lending is truly accessible to everyone - including the poor, unbanked and small businesses. Whilst less collateral may be required if/when cryptocurrencies stop being volatile, for now the amount required before lending makes it inaccessible to the unbanked.

Second, cryptocurrency lending and DeFi allows holders to engage in investment activities as they can borrow to invest in more cryptocurrencies using collateral which can also increase in value. However, there is no guarantee that this would be a profitable investment given that a lot of collateral is committed to borrow cryptocurrency whose value has had a history of volatility and has resulted in huge losses for investors.

Third, cryptocurrency lending DeFi platforms are interoperable (in that they can work with other decentralised applications). This provides more options when taking out loans or lending crypto. For example, after taking out a DAI loan from MakerDAO, users can convert it to other cryptocurrencies to gain leverage, creating different possibilities to further invest.

Fourth, the system is deemed as efficient and cheaper as everything is built on blockchain and there are no charges for banking/ intermediary services since everything is peer-to-peer driven. Furthermore, the system is more dynamic, resilient and transparent as DApps are open-source and interoperable enabling developers to upgrade and develop more projects leading to the provision of more valuable DeFi services that contribute to the ecosystem.

\section{LIMITATIONS OF DeFi}

Despite the benefits, DeFi has clear limitations. The strongest of these is the on-ramps and off-ramps challenge. This is the challenge of changing fiat currency into crypto and crypto into fiat. Although DeFi claims to be a form of non-custodial finance, it is difficult to find a DeFi platform with a non-custodial solution that allows a user to get in and out of crypto in a non-custodial way. All the current $\mathrm{DeFi}$ platforms appear to be crypto to crypto and although they provide the ability to save and earn interest on users' money on a platform such as compound finance, which is a platform that enables users to deposit their crypto and earn interest on it, users are unable to get fiat funds directly in their fiat bank account. It is, as such, difficult to use the earnings on interest in the real economy. The most convenient, but also complex, way to convert crypto back to fiat currency is through a centralised exchange. However, users are able to acquire cryptocurrencies through their non-custodial wallet directly using their private keys or through services such as LocalBitcoins.com, where unbanked persons are able to trade their local currency for crypto and vice versa by simply doing a real-world peer-to-peer exchange. (https://www.webcitation.org/6n8YgGQ51?url=http:// gizmodo.com/the-street-dealers-who-peddle-bitcoin-1683847723). So, although difficult, it is possible to transact within the DeFi 
space and convert crypto to fiat currency without using a centralised exchange but this is not always convenient.

It should also be mentioned that there is a new technology which uses a non-custodial platform to get in and out of crypto or fiat that is being developed by Nimiq - an open-source decentralised peer-to-peer payment protocol for the world wide web. Nimiq is working on a technology which is coming out this year, which is a fiat smart contract. This is called Nimiq OASIS and it would enable noncustodial crypto-fiat trades. This is being built in collaboration with Ten 31 Bank owned by a German bank, WEG bank, and the technology aims to make fiat currency programmable as if they were tokens on a blockchain (https://cryptoadventure.org/nimiq-coinreview-2020-why-it-could-be-a-hidden-gem/). Once Nimiq OASIS is launched, it would offer a non-custodial solution that allows a user to get in and out of crypto without the use of a centralised exchange or custodian wallet provider.

Although the intersection of DeFi with the real economy or fiat economy currently mainly through regulated centralised exchanges provides a point of regulation even for $\mathrm{DeFi}$, the challenges of enforcing FATF Recommendation 16 (that it is only as effective as its effective implementation in all countries, as discussed above) is a potential limitation for regulating DeFi.

\section{RISKS OF DeFi}

Although DApps are not well-known or used by the public their usage is indeed growing. Over the past 20 months, crypto lending has grown significantly and according to Graychain's first report (The Crypto Credit Report (Q2 2019) issued in August 2019) on the collateralised crypto lending industry, approximately 244,000 loans have been originated and an estimated value of $\$ 4.7 \mathrm{bn}$ has been lent out over the history of the sector. Also, according to DeFi Pulse (https://defipulse. $\mathrm{com} /$ ) the best tracker of developments in the market the current amount locked into DeFi as at 9 April 2020 is $\$ 738 \mathrm{~m}$.

The growth in the industry coupled with the fact that $\mathrm{DeFi}$ and crypto lending operates on a largely decentralised basis raises a few regulatory concerns which need to be addressed not only at domestic levels but also at the international level.

First, DeFi crypto lending can increase financial stability risks. Despite being described as decentralised, DeFi crypto lending facilitates the concentration of risk previously in centralised financial institutions to predominantly software developers. This is because many activities on blockchains/DLTs, such as control over source code and code development, ownership of the assets, operation of the infrastructure and crypto-assets mining, are predominantly in the hands of software developers, according to De Filippi and Wright in Blockchain and Law, p 173.

Second, DeFi also raises governance, legal and operational risks issues as in typically decentralised permissionless platforms, it becomes difficult to hold any particular person or entity accountable for any technological failure that may result in the collapse of the system. It may also be challenging to allocate liability in transactions involving anonymous parties in smart contracts - especially when used to disguise fraudulent activities. As reported by De Filippi and Wright in Blockchain and Law, p 200, smart contracts can contain bugs. There have been occasions where bugs have occurred and users have lost their funds by having them stuck in the smart contracts. This is a risk that people are taking by participating in the DeFi ecosystem.

Third, DeFI could lead to regulatory arbitrage at two levels. First, as DeFi transactions are typically cross-border, as and when regulatory standards are established for this sector, platforms may gravitate towards jurisdictions with no/less regulatory requirements. Related to this is the challenge of global coordination, especially as jurisdictions are at varying degrees of financial regulatory advancement. So, while some would naturally embrace regulatory technology solutions (RegTech) for regulating decentralised financial technologies (some of which have been discussed by De Filippi and Wright in Blockchain and Law, p 196), other jurisdictions may find it difficult to implement RegTech standards and solutions such as for example, those designed to regulate crypto lending and other financial services occurring on DeFi platforms.

Regulatory arbitrage could also emanate from the existence of dual financial systems in a domestic setting - real and virtual - which, if providing the same services, can potentially lead to a total transfer of financial services to a virtual system where regulation has limited reach. This, of course, would have wider economic implications on the ability of governments and central banks to make necessary interventions in stabilising economies as and when required.

\section{POLICY ISSUES}

The analysis above highlights two issues:

- A wholistic approach to regulating the crypto industry is required: This approach should take on board the regulation of crypto transactions occurring in centralised exchanges (or custodial platforms) and those crypto transactions occurring on decentralised platforms (or non-custodial platforms). FATF Recommendation 16 clearly does not provide for the latter so there is a gap.

- Which regulatory approach should be adopted? Traditional finance regulates institutions as opposed to users or bank customers. Within decentralised finance, it would appear that a change of focus is required. The approach could either focus on regulating the developers of decentralised applications or platform providers or possibly the users of these platforms or perhaps both. Whichever approach is adopted would require certain parameters to be drawn. Would platform developers and providers be required to know the identity of those transacting on them - such as is now required for centralised exchanges or should they be made responsible for nefarious activities transacted by users of their platforms? If users were targeted, what would they be held responsible for? Would they be required to fulfil some sort of digital identity and should they be required to know the identity of the parties they are transacting with? Of course, all of this would have implications for accessibility to DeFi services, the absence of which, promoters have argued, is 
what facilitates financial inclusion across the world. It would, of course be necessary to strike the right balance between attaining financial inclusion and preventing financial crime which could have international security, financial and economic implications should DeFi continue to grow globally.

- Open source decentralised blockchains cannot be shut down: If, according to De Filippi and Wright, Blockchain and the Law, p 144, the Ethereum blockchain which is a tamper-resistant, resilient blockchain enabling the execution of autonomous smart contract code cannot be shut down for as long as you have developers working on it and users using it, to what extent can decentralised finance applications built on it be regulated or completely shut down? If regulators cannot shut down the underlying code upon which platforms are built, in the way that they can withdraw the licence of a bank or other financial institution, how effective can the regulation of these activities be? This raises the question of what the most pragmatic approach should be for regulating activities occurring within the $\mathrm{DeFi}$ eco system? It would appear that the most pragmatic approach to regulation should embrace collaboration with all necessary stakeholders. Regulators should be willing to engage with a wider group of stakeholders, including academia, businesses, software developers, the engineering community, investors, consumers and users. This would facilitate efficient evaluation of the risks and opportunities of decentralised finance and technologies and help achieve a balanced approach to regulation which does not stifle innovation.

\section{CONCLUSION}

According to DeFI Pulse, the current amount locked in the DeFi space is $\$ 738 \mathrm{~m}$ which is not a significant amount that can rock the international financial system. However, with growth in the industry set to rise given its potential to reach the unbanked, it should be monitored by regulators. Furthermore, as stated above, according to Graychain's report on the collateralised crypto lending industry in August 2019, approximately 244,000 loans have been originated and an estimated value of $\$ 4.7 \mathrm{bn}$ has been lent out over the history of the sector which further buttresses the need for regulatory oversight.

Also approaches to mitigating the potential gaps in FATF Recommendation 16 with respect to centralised exchanges should be put on the agenda of the FATF and other international standard setters. Linked to this is a much-needed plan for a wholistic approach to regulating the industry - covering both decentralised and centralised platforms for crypto transactions. Finally, given that decentralised platforms cannot truly be shut down, it is time for regulators to realise that they can hardly adopt a robust regulatory regime without industry cooperation.

\section{Further Reading:}

- The role of depositaries in safekeeping digital assets: the fact rather than the fiction (2020) 5 JIBFL 319.
Legal and regulatory issues for custodians and administrators of digital assets (2019) 1 JIBFL 35.

- LexisPSL: Financial Services Practice Note: Financial regulation of cryptoassets. 